\title{
Travelling fronts for multidimensional nonlinear transport equations
}

\author{
by \\ Hartmut R. SCHWETLICK ${ }^{1}$ \\ Mathematik, ETH-Zentrum, CH-8092 Zürich, Switzerland \\ Manuscript received 27 September 1999, revised 18 April 2000
}

ABSTRACT. - We consider a nonlinear transport equation as a hyperbolic generalisation of the well-known reaction-diffusion equation. We show the existence of strictly monotone travelling fronts for the three main types of the nonlinearity: the positive source term, the combustion law, and the bistable case.

In the first case there is a whole interval of possible speeds containing its strictly positive minimum. For subtangential nonlinearities we give an explicit expression for the minimal wave speed.

(C) 2000 L'Association Publications de l'Institut Henri Poincaré. Published by Elsevier B.V. All rights reserved

RÉSUMÉ. - Nous considérons une équation de transport nonlinéaire comme étant une généralisation hyperbolique de l'équation de réactiondiffusion bien connue. Nous montrons l'existence de fronts progressifs strictement monotones pour les trois principaux types de la nonlinéarité : le terme source positif, la loi de combustion et le cas «bistable». Dans le premier cas il existe tout un interval de vitesses possibles comprenant son minimum. Dans le cas de nonlinéarités «sous-tangentielles» nous donnons une expression explicite de cette vitesse minimale.

(C) 2000 L'Association Publications de l'Institut Henri Poincaré. Published by Elsevier B.V. All rights reserved

\footnotetext{
${ }^{1}$ E-mail: hartmut.schwetlick@ math.ethz.ch.
} 


\section{INTRODUCTION}

The work of Fisher [7] and Kolmogorov, Petrovsky and Piskounov [11] inspired the study of the asymptotic behaviour of spreading and interacting particles on unbounded domains. Both articles modelled spread and interaction by a reaction-diffusion equations. In particular it was shown that suitable initial configurations converge asymptotically to travelling front solutions. This observation lead Aronson and Weinberger in a series of papers to introduce the concept of the asymptotic speed of propagation, cf. $[2,1,15,3]$. These articles already include the treatment of integral equations as well as discrete time and space models. The idea and the computation of the asymptotic speed was then extended to more general integral equations in $[6,14,13,16]$.

The parabolic nature of the reaction-diffusion equation leads to the unrealistic phenomenon of unbounded particle speeds. Motivated by the theory of diffusive transport we shall therefore propose a hyperbolic generalisation, which arises if one replaces Brownian motion by a transport process. Since the state space is enlarged by the set of possible velocities/directions, the implementation of the reaction terms may differ from the reaction-diffusion case.

Our goal is to show the existence of travelling front solutions of the following hyperbolic equation. For the density $U=U(t, x, v)$ of particles, moving at $(t, x) \in \mathbb{R}_{+} \times \mathbb{R}^{n}$ with normalised velocity $v \in V \subset$ $\bar{B}_{1}\left(0 ; \mathbb{R}^{n}\right)$, we consider the nonlinear transport equation

$$
\left(\partial_{t}+\gamma v \cdot \nabla+\mu L\right) U=\frac{1}{|V|} M(\bar{U})+G(\bar{U}) U \quad \text { in } \mathbb{R}_{+} \times \mathbb{R}^{n} \times V .
$$

Here $\gamma$ denotes the maximal particle speed, $\bar{U}(t, x)=\int_{V} U(t, x, v) \mathrm{d} \sigma_{v}$ is the local total density, weighted by a positive measure $\sigma$ on $V$, having bounded volume $|V|=\int_{V} \mathrm{~d} \sigma_{v}$. We assume that $\sigma$ and $V$ are rotationally symmetric. Turning of particles is governed by a Poisson process with parameter $\mu$ according to the operator $L U=U-\frac{1}{|V|} \bar{U}$. The operator $L$ describes the local deviation from the mean and provides the diffusivity of the transport process. Physically speaking $L$ defines isotropic scattering. The reaction is modelled by a uniformly distributed production $M \geqslant 0$, depending on the total density $\bar{U}$, and a mass action law with rate $G$, which acts on the individual density $U$. Note that all annihilation processes must be modelled by a rate to preserve positivity. Hence they are contained in $G$. We call $f(z)=M(z)+G(z) z$ the 
net reaction. It is this net effect, which has to be compared with the nonlinearity used in reaction-diffusion equations. In our modell particles react not according to their individual velocities, which is reasonable in certain chemical reactions or in biological modelling, see also [12, Section 3.1]. The prototypical organisms whose motion can be described by a transport equation are flagellated bacteria, the best studied of which is E. coli. More examples can be found in $[12,10]$, including the locomotion of mouse fibroblasts and crawling caterpillars.

The isotropic modelling of the reaction in (1) is in contrast to the Boltzmann as well as to the neutron transport equation. In the former case post- and pre-collision velocities obey deterministic relations, whereas interactions are less relevant in the latter case.

Throughout the paper we make the following assumptions on $f$ (i.e., on $M$ and $G$ ):

(H1) The functions $M$ and $G$ are defined and Lipschitz continuous on $[0,1]$, satisfying $M(0)=0$ and $M(1)+G(1)=0$. Hence, $f$ is defined and Lipschitz continuous on $[0,1]$ with $f(0)=f(1)=$ 0 . Furthermore we distinguish three main types:

(A) $f>0$ on $(0,1)$.

(B) There is a $\theta \in(0,1)$ such that $f \equiv 0$ on $[0, \theta]$ and $f>0$ on $(\theta, 1)$.

(C) There is a $\theta \in(0,1)$ such that $f<0$ on $(0, \theta)$ and $f>0$ on $(\theta, 1)$.

Type $\mathrm{A}$ is the so-called positive source term reaction and includes the famous nonlinearities $z(1-z)$ and $z(1-z)^{2}$ used by Fisher [7] and Kolmogorov, Petrovsky and Piskounov [11], respectively. In the present transport context we propose for the RHS of (1) the function $(1-\bar{U})^{k} U$, i.e. $M \equiv 0, G=(1-z)^{k}$, as a suitable implementation of the mass action law. Type B can be found in many models of combustion theory, where the burning reaction is triggered by an ignition temperature. Type $\mathrm{C}$ refers to the bistable reaction law, since $f$ admits two stable equilibria.

We comment on the various reaction types. The classical results in [3] for the reaction-diffusion equation show that for type A there are fronts for all speeds greater or equal to a uniquely defined minimal speed. On the other hand, for type $\mathrm{C}$ there is only a single front, unique up to translation, which connects the equilibria 0 and 1 . Berestycki and Larrouturou [4] proved also for type B the uniqueness of the front. Hadeler [9] considered fronts for an one-dimensional transport equation where only the two velocities $-\gamma$ (left) and $+\gamma$ (right) are admitted. The resulting two component system for the travelling front equation was 
shown to be equivalent (up to scaling) with the classical one in [3] coming from the reaction-diffusion equation. For type A there is an interval of positive speeds containing in particular all large speeds up to the maximal particle speed $\gamma$, while type $\mathrm{C}$ leads again to a unique front. Note that the hyperbolicity excludes any speed exceeding $\gamma$.

\section{The travelling wave equation}

We are concerned with the existence of travelling plane wave solutions of (1), also called fronts, i.e., of solutions of the form

$$
U(t, x, v)=u(\tau, v), \quad \tau=x \cdot \eta-c t,
$$

where the unit vector $\eta$ stands for the direction along which the wave is propagating with speed $c \in(-\gamma, \gamma)$. Furthermore, we require $u \geqslant 0$, $\bar{u} \in[0,1]$ and the asymptotic boundary conditions

$$
u(-\infty, v) \equiv \frac{1}{|V|}, \quad u(+\infty, v) \equiv 0
$$

This says, that the total density along the wave connects the two distinguished equilibria of $f$ through the phase space region $\bar{u} \in[0,1]$. Note that the rotational symmetry of the problem allows one to fix $\eta=e_{1}$. Inserting this ansatz into (1) we are lead to the question whether or not there is a solution $(c, u)$ of the stationary problem

$$
\left(\left(\gamma v_{1}-c\right) \partial_{\tau}+\mu L\right) u=\frac{1}{|V|} M(\bar{u})+G(\bar{u}) u \quad \text { in } \mathbb{R} \times V
$$

which also satisfies (2). Setting $c=\gamma \kappa$ we introduce a normalised speed $\kappa \in(-1,1)$. Provided that $\mu>G$ on $[0,1]$ we can rescale $\tau \in \mathbb{R}$ by the positive factor $\gamma /(\mu-G(\bar{u}))$. Denoting the new variable by $t$ and the derivative with respect to $t$ by a dot we arrive at the principal form of (2),(3)

$$
\begin{aligned}
& \left(v_{1}-\kappa\right) \dot{u}+u=\frac{1}{|V|} F(\bar{u}) \quad \text { in } \mathbb{R} \times V, \\
& u(-\infty, v) \equiv \frac{1}{|V|}, \quad u(+\infty, v) \equiv 0, \quad u \geqslant 0, \bar{u} \in[0,1],
\end{aligned}
$$


now involving a $v$-independent, also called isotropic, source term

$$
F(z)=z+\frac{f(z)}{\mu-G(z)} .
$$

We remark that the speed $\kappa$ together with the solution $u$ are considered as unknowns. We refer to $u$ as the shape function. In the following the function $F$ will incorporate all relevant features of the reaction into our wave problem. Therefore, we restate the various reaction types for $F$ : (A) $F>\operatorname{Id}$ on $(0,1)$; (B) There is $\theta \in(0,1)$ with $F=\operatorname{Id}$ on $[0, \theta], F>\mathrm{Id}$ on $(\theta, 1)$; (C) There is $\theta \in(0,1)$ with $F<\operatorname{Id}$ on $(0, \theta), F>\operatorname{Id}$ on $(\theta, 1)$.

To construct solutions to (4) we consider the limit of solutions on finite cylinders $(-R,+R) \times V$. The method is inspired by the work of Berestycki and Nirenberg [5] on travelling fronts of reaction diffusion equations in cylinders. To prove uniqueness of solutions on finite cylinders we use an adaption of the sliding domain method, originally introduced in [5] for elliptic equations. This method crucially relies on a maximum principle which is available in the elliptic case. We are able to use the power of the sliding method also for stationary transport equations of the form (4) if we require the following monotonicity assumption on the nonlinearity.

(H2) $\mu>G$ and the function $F$ is strictly increasing on $[0,1]$.

We include the inequality $\mu>G$ because it is needed to derive (4) and it ensures that $F$ is well-defined. Note that $\mu>G$ is always satisfied if $G$ contains no gain term. Also the second part of the hypothesis requires, that the hyperbolic diffusion in (1), measured by the parameter $\mu$, is sufficiently strong. This can be seen by the fact that if $f$ and $G$ are $C^{1}$ functions then (H2) is implied by the inequality

$$
\frac{\mathrm{d}}{\mathrm{d} z} \frac{f(z)}{\mu-G(z)}>-1 .
$$

The existence of solutions follows classical steps, once we know monotonicity and compactness properties of a solution operator related to (4). We will establish suitable compactness properties by applying the regularity results for velocity averages of Golse, Lions, Perthame and Sentis [8]. Hence, we need to require the main assumption therein:

(H3) There are constants $C \geqslant 1, \alpha \in(0,1)$, such that the measure $\sigma$ on $V$ satisfies

$$
\frac{1}{|V|}\left|\left\{v \in V:\left|v_{1}-\kappa\right|<\varepsilon\right\}\right| \leqslant C \varepsilon^{\alpha} \quad \text { for all } \kappa \in[-1,1] .
$$


We remark that the uniform measure on the sphere $S^{n-1}$ in $\mathbb{R}^{n}$ satisfies (H3) for any $\alpha \in(0,1 / 2]$ in case $n=2$ and $\alpha \in(0,1)$ for all $n \geqslant 3$.

\section{Results}

THEOREM 1.1. - Assuming type A there exists a minimal wave speed $\kappa^{*} \in(0,1)$, such that Eq. (4) has a solution $(\kappa, u)$ if and only if $\kappa \in$ $\left[\kappa^{*}, 1\right)$. For any $\kappa \in\left[\kappa^{*}, 1\right)$ there is a solution with a strictly decreasing shape function.

THEOREM 1.2. - Assume type $B$ or $C$. There exists a speed $\kappa^{*} \in$ $(-1,1)$, such that for $\kappa=\kappa^{*}$ Eq. (4) has a solution $(\kappa, u)$. The shape $u$ is strictly decreasing in $t$. There is a speed $\bar{\kappa} \in(0,1)$ such that $(4)$ has no solution $(\kappa, u)$ for any $\kappa>\bar{\kappa}$.

Note that the arguments deriving (4) imply, that any solution $(\kappa, u)$ of (4) is equivalent with a travelling wave solution $(c, U)$ of (1) having speed $c=\gamma \kappa$ and the desired asymptotic behaviour.

In Section 2 we show that (4) can be transformed into a scalar integral equation for the total density $\bar{u}$ alone. Using (H3) we obtain as a preliminary result that the shape function $u(t, v)$ of any solution $(\kappa, u)$ of (4) is uniformly continuously differentiable in $t$, provided $v_{1} \neq \kappa$. Despite this defect in the dependence on $v$ the total density $\bar{u}$ is still uniformly continuously differentiable. Then Theorem 1.1 is proved by an adaption of the methods developed by Weinberger [16] for the treatment of very general integral problems of type A. However, Weinberger's results do not lead to an existence proof of fronts for the types B or C. Section 2 concludes with an explicit variational description of the minimal wave speed for subtangential type-A-reactions.

Theorem 1.2 is proved in Section 3. We will construct a front by considering (4) on a finite cylinder $(-R, R) \times V$ and then let $R$ go to infinity. This idea was used by Berestycki and Nirenberg [5] to show the existence of front-like solutions of elliptic equations for nonlinearities of type $\mathrm{A}, \mathrm{B}$ or $\mathrm{C}$. In [5] it is assumed that $f$ has Hölder continuous derivatives at 0 and 1 , in particular $f^{\prime}(1)<0$, to prove that the constructed solution obeys the asymptotic boundary condition. Furthermore, the assumption is needed in the proof for type A to show that one can indeed construct fronts of minimal speed. However, using Weinberger's approach we can prove Theorem 1.1, i.e. type A, without any further assumptions on $f$. Subsequently, Theorem 1.1 is used in the proof of Theorem 1.2 to treat also the types $\mathrm{B}$ or $\mathrm{C}$ without further assumptions on $f$. 
No uniqueness of the speed or the shape of the front is proven for the types B and C. This is subject to the author's current research, which includes the stability of these fronts. Furthermore, it is well-known that for $\mu \rightarrow \infty$ the transport process approximates brownian motion. A natural question arising here is, if the fronts of the transport equation converge to the fronts for the limiting reaction-diffusion problem.

\section{TYPE A - THE POSITIVE SOURCE TERM}

For the convenience of the reader we start with a brief exposition of Weinberger's results in [16]. Let $W=C^{0}(\mathbb{R} ;[0,1])$ and $Q: W \rightarrow W$ an operator satisfying

(1) $Q[0]=0, Q[1]=1$.

There is a $\theta \in[0,1)$ such that $Q[\theta]=\theta$ and $Q[z]>z$ for all $z \in(\theta, 1)$.

(HQ) (2) $Q \circ S_{c}=S_{c} \circ Q$ for all $c \in \mathbb{R}$.

(3) If $w_{1} \leqslant w_{2}$ then $Q\left[w_{1}\right] \leqslant Q\left[w_{2}\right]$.

(4) $Q\left[w_{n}\right] \rightarrow Q[w]$ pointwise if $w_{n} \rightarrow w$ uniformly on bounded sets (ubs.).

(5) $\left\{Q\left[w_{n}\right]\right\}$ has a ubs.-convergent subsequence for any sequence $\left\{w_{n}\right\}$.

Weinberger views $Q$ as the time-1-evolution operator of a general dynamical system. The first condition on the flow operator $Q$ reflects the assumptions in (H1) on the nonlinearity $f$ : while $\theta=0$ corresponds to type $\mathrm{A}, \theta>0$ contains reactions of type $\mathrm{B}$ and $\mathrm{C}$. The other conditions state that $Q$ is translation invariant, monotone, continuous, and compact.

We call $w \in W$ a travelling wave solution of $Q$ with speed $c \in \mathbb{R}$, if

$$
\begin{gathered}
w=Q \circ S_{c}[w], \\
w(-\infty)=1 \geqslant w(t) \geqslant 0=w(\infty),
\end{gathered}
$$

where $S_{c}: W \rightarrow W$ denotes the shift operator, i.e. $S_{c}[w](t):=w(t+c)$.

Let $\phi \in W$ nonincreasing and satisfy

$$
\phi(-\infty) \in(\theta, 1), \quad \phi(t)=0 \quad \text { for } t \geqslant 0 .
$$


For any $c \in \mathbb{R}$ the sequence

$$
w_{n+1}(c, t)=\max \left\{\phi(t), Q \circ S_{c}\left[w_{n}\right](t)\right\}, \quad w_{0}(c, t)=\phi(t)
$$

is nondecreasing and uniformly bounded in $n$, nonincreasing and continuous in $c, t$. Therefore, $w(c, t)=\lim _{n} w_{n}(c, t)=\sup _{n} w_{n}(c, t)$ is a uniquely defined function which is nonincreasing and lower semicontinuous in $c, t$. Set

$$
c^{*}:=\sup \{c: w(c, t) \equiv 1\} .
$$

THEOREM 2.1 ([16, Theorem 6.6]). - The number $c^{*}$ is independent of the choice of the initial function $\phi$.

(a) For all $c<c^{*}$ there is no travelling front solution with speed $c$.

(b) If $\theta=0$ then there exists a nonincreasing travelling front for any speed $c, c \geqslant c^{*}$.

Let us remark that in case A, i.e., $\theta=0$, the number $c^{*}$ is indeed the minimal speed of fronts for $Q$. Note that nothing is said about the existence of fronts for $\theta>0$.

Actually, Weinberger did not show (a) but it is an immediate consequence from the following result which he used to prove that $c^{*}$ is independent of $\phi$.

Proposition 2.2 ([16, Lemma 5.3, Proposition 5.1]). - Let $c \in \mathbb{R}$, $\phi \in W$ a nonincreasing function satisfying $(7)$, and $w_{n}(c, t)$ the sequence (8). Then the following are equivalent:

(i) $c<c^{*}$;

(ii) $w_{n}(c, t) \rightarrow 1$;

(iii) There exists $n \in \mathbb{N}$ such that $w_{n}(c, 0)>\phi(-\infty)$.

Proof of Theorem 2.1(a). - Assume that for $c<c^{*}$ a front $w_{c} \in W$ exists satisfying (6). Since $w_{c}(-\infty)=1$ we can assume that $w_{c}(0) \in$ $(\theta, 1)$ and $w_{c}(t)>w_{c}(0)$ for all $t<0$. Consider the sequence $w_{n}(c, t)$ constructed from a $\phi$ satisfying $\phi(-\infty)=w_{c}(0)$. Clearly

$$
w_{0}(c, t)=\phi(t) \leqslant w_{c}(t)
$$

and from (HQ3) we infer $w_{n}(c, t) \leqslant w_{c}(t)$. But Proposition 2.2 states that there must be a number $n \in \mathbb{N}$ such that $w_{n}(c, 0)>\phi(-\infty)=w_{c}(0)$, which is impossible. 
Weinberger calculates also bounds for $c^{*}$ for certain operators $Q$.

THEOREM 2.3 ([16, Theorems 6.3, 6.4]). -

(i) If $Q$ satisfies $Q[w](t) \leqslant \int_{\mathbb{R}} K(s-t) w(s) \mathrm{d} s$, then

$$
c^{*} \leqslant \inf _{\lambda>0} \frac{1}{\lambda} \log \int_{\mathbb{R}} \mathrm{e}^{-\lambda s} K(s) \mathrm{d} s .
$$

(ii) If there is a kernel $L$ with $\int_{\mathbb{R}} L(s) \mathrm{d} s>1$ and a positive $\varepsilon$ such that

$$
Q[w](t) \geqslant \int_{\mathbb{R}} L(s-t) w(s) \quad \text { for any } w \in W \text { with } 0 \leqslant w \leqslant \varepsilon,
$$

then

$$
c^{*} \geqslant \inf _{\lambda>0} \frac{1}{\lambda} \log \int_{\mathbb{R}} \mathrm{e}^{-\lambda s} L(s) \mathrm{d} s .
$$

Note that for $\int_{\mathbb{R}} L(s) \mathrm{d} s<1$ the lower bound would be trivial, i.e., equal to $-\infty$.

\subsection{The integral equation}

We return to our travelling front equation (4). To apply Weinberger's results we will derive an integral formulation of (4) which is in fact an integral equation for the total density $\bar{u}$ alone. To this aim consider for any $\phi \in W$ the linear, inhomogeneous problem to (4)

$$
\left(v_{1}-\kappa\right) \dot{u}+u=\frac{1}{|V|} F(\phi), \quad \text { in } \mathbb{R} \times V .
$$

We have an explicit description of the solution $u=T_{\kappa} \circ \mathcal{F}[\phi]$. Here $\mathcal{F}[\phi](t):=F(\phi(t))$ denotes the Nemytskij operator on $W$, whereas

$$
\begin{gathered}
T_{\kappa}: L^{\infty}(\mathbb{R}) \rightarrow L^{\infty}\left(V ; L^{\infty}(\mathbb{R})\right), \\
T_{\kappa}[\psi](t, v):=\frac{1}{|V|} \int_{-\infty}^{0} \mathrm{e}^{s} \psi\left(t+\left(v_{1}-\kappa\right) s\right) \mathrm{d} s
\end{gathered}
$$

is the solution operator for the linear problem on unbounded domains with an isotropic inhomogeneity $\left(v_{1}-\kappa\right) \dot{u}+u=\frac{1}{|V|} \psi(t)$ in $\mathbb{R} \times V$. 
Now, integration over $V$ gives the solution operator for the total density

$$
\bar{u}=Q_{\kappa}[\phi]:=\overline{T_{\kappa} \circ \mathcal{F}[\phi]}=\bar{T}_{\kappa} \circ \mathcal{F}[\phi],
$$

where

$$
\bar{T}_{\kappa}: L^{\infty}(\mathbb{R}) \rightarrow L^{\infty}(\mathbb{R}), \quad \bar{T}_{\kappa}[\psi](t):=\int_{\mathbb{R}} K_{\kappa}(s-t) \psi(s) \mathrm{d} s
$$

is a convolution with positive probability kernel

$$
K_{\kappa}(s)=\frac{1}{|V|} \int_{v \in V, \frac{s}{v_{1}-\kappa}<0} \frac{\mathrm{e}^{\frac{s}{v_{1}-\kappa}}}{\left|v_{1}-\kappa\right|} \mathrm{d} \sigma_{v},
$$

i.e., it satisfies $K_{\kappa}>0$ and $\int_{\mathbb{R}} K_{\kappa}(s) \mathrm{d} s=1$.

In contrast to the integral equation coming from the dynamical problem (1), $Q_{\kappa}$ enjoys a good compactness property: Fix $\psi \in L^{\infty}$ and define $u:=T_{\kappa} \psi$. Recalling the definition of $T_{\kappa}$ we obtain $|u|_{L^{\infty}(\mathbb{R} \times V)} \leqslant$ $\frac{1}{|V|}|\psi|_{L^{\infty}(\mathbb{R})}$. Furthermore, $\left|\left(v_{1}-\kappa\right) \dot{u}\right|_{L^{\infty}(\mathbb{R} \times V)} \leqslant \frac{2}{|V|}|\psi|_{L^{\infty}(\mathbb{R})}$ holds. Hence, $[8$, Lemma 7] yields the following

LEMmA 2.4. - The hypothesis (H3) implies

$$
\left|\bar{T}_{\kappa} \psi\right|_{C_{b}^{\alpha}(\mathbb{R})} \leqslant C_{0}|\psi|_{L^{\infty}(\mathbb{R})},
$$

where $C_{0}$ only depends on $C, \alpha$. Furthermore, $\bar{T}_{\kappa}$ respects the upper and lower bounds, i.e.

$$
\inf \psi \leqslant \inf \bar{T}_{\kappa} \psi \leqslant \sup \bar{T}_{\kappa} \psi \leqslant \sup \psi .
$$

As usual, the Hölder norm is defined by

$$
|\phi|_{C_{b}^{\alpha}(\mathbb{R})}:=|\phi|_{L^{\infty}(\mathbb{R})}+\sup _{t \neq t^{\prime}} \frac{\left|\phi(t)-\phi\left(t^{\prime}\right)\right|}{\left|t-t^{\prime}\right|^{\alpha}} .
$$

From (H2) we know that $\mathcal{F}$ maps $W$ into itself, also continuously by (H1). Therefore the above lemma implies that $Q_{\kappa}$ is a continuous and compact mapping of $W$ into itself.

We show now, that any solution of (4) is equivalent to a fixed point $\bar{u} \in$ $W$ of the corresponding integral equation for the total density satisfying 
in addition the asymptotic boundary condition, i.e.,

$$
\begin{gathered}
\bar{u}=Q_{\kappa}[\bar{u}], \\
\bar{u}(-\infty)=1 \geqslant \bar{u}(t) \geqslant 0=\bar{u}(\infty) .
\end{gathered}
$$

Clearly, if $u$ is a solution of (4) then $\bar{u}$ satisfies (9). Since $\bar{u} \in C_{b}^{\alpha}(\mathbb{R})$ by Lemma 2.4 , we have $\bar{u} \in W$. For the opposite direction recall that for a given solution $\bar{u}$ of (9) the function $u=T_{\kappa} \circ \mathcal{F}[\bar{u}]$ obeys the differential equation in (4). We need to verify the correct asymptotics. Since $\bar{u} \in W$ and $\bar{u}(\infty)=0$ we find for any $\varepsilon>0$ a number $M \in \mathbb{R}$ such that $\mathcal{F}[\bar{u}](s)<\varepsilon$ for $s>M$. Futhermore, $0 \leqslant \mathcal{F}[\bar{u}] \leqslant 1$ by the monotonicity of $F$. Let $N:=\log \varepsilon^{-1}$. For $t \geqslant M+(1-\kappa) N$ we obtain

$$
\begin{aligned}
0 \leqslant T_{\kappa} \circ \mathcal{F}[\bar{u}](t, v) & =\frac{1}{|V|} \int_{-\infty}^{0} \mathrm{e}^{s} F\left(\bar{u}\left(t+\left(v_{1}-\kappa\right) s\right)\right) \mathrm{d} s \\
& \leqslant \frac{1}{|V|} \int_{-N}^{0} \mathrm{e}^{s} \varepsilon \mathrm{d} s+\frac{1}{|V|} \int_{-\infty}^{-N} \mathrm{e}^{s} \mathrm{~d} s \\
& <\frac{1}{|V|}\left(\varepsilon+\mathrm{e}^{-N}\right)=\frac{2}{|V|} \varepsilon .
\end{aligned}
$$

A similar proof is used for $t \rightarrow-\infty$.

We will use the following simple observation: Any solution of (9) is equivalent to a speed 0 front of $Q_{\kappa}$. Let us remark that $Q_{\kappa}$ is the solution operator of the stationary problem for a fixed nonlinear inhomogeneity and NOT the time-1-evolution operator of the dynamical problem (1) in the moving coordinate system $(t, x+\kappa t)$. Hence, fronts for $Q_{\kappa}$ with nonzero speed have no particular physical meaning.

In the remainder of this paragraph we will verify the assumtions (HQ) for $Q_{\kappa}$.

A slight modification of the proof for [8, Lemma 7] gives

LEMMA 2.5. - Assume (H3). There is a constant $C_{1}=C_{1}(C, \alpha)$ such that

$$
\left|\bar{T}_{\kappa} \psi\right|_{C_{b}^{\beta+\alpha(1-\beta)}(\mathbb{R})} \leqslant C_{1}|\psi|_{C_{b}^{\beta}(\mathbb{R})} \quad \text { for all } \beta \in(0,1) .
$$

Proof. - Set $u:=T_{\kappa} \psi, \psi \in C_{b}^{\beta}(\mathbb{R})$. From $\left|\left(v_{1}-\kappa\right) \dot{u}\right|_{L^{\infty}(\mathbb{R} \times V)} \leqslant$ $\frac{2}{|V|}|\psi|_{L^{\infty}(\mathbb{R})}$ follows 


$$
\begin{aligned}
\left|\bar{u}(t)-\bar{u}\left(t^{\prime}\right)\right| \leqslant & \int_{\left|v_{1}-\kappa\right|<\delta}\left|u(t, v)-u\left(t^{\prime}, v\right)\right| \mathrm{d} \sigma_{v} \\
& +\int_{\left|v_{1}-\kappa\right|>\delta}\left|\int_{t^{\prime}}^{t}\left(v_{1}-\kappa\right) \dot{u}(s, v) \mathrm{d} s\right| \frac{\mathrm{d} \sigma_{v}}{\left|v_{1}-\kappa\right|} \\
\leqslant & |\psi|_{C_{b}^{\beta}(\mathbb{R})}\left[\left|t-t^{\prime}\right|^{\beta} \frac{1}{|V|} \int_{\left|v_{1}-\kappa\right|<\delta} \mathrm{d} \sigma_{v}\right. \\
& \left.+2\left|t-t^{\prime}\right| \frac{1}{|V|} \int_{\left|v_{1}-\kappa\right|>\delta} \frac{\mathrm{d} \sigma_{v}}{\left|v_{1}-\kappa\right|}\right] .
\end{aligned}
$$

From (H3) we infer $\frac{1}{|V|} \int_{\left|v_{1}-\kappa\right|<\delta} \mathrm{d} \sigma_{v} \leqslant C \delta^{\alpha}$. As in the proof of [8, Lemma 7] we find

$$
\frac{1}{|V|} \int_{\delta<\left|v_{1}-\kappa\right|} \frac{\mathrm{d} \sigma_{v}}{\left|v_{1}-\kappa\right|} \leqslant \frac{2 C}{1-\alpha} \delta^{\alpha-1} .
$$

Finally, we set $\delta=\frac{4 C}{1-\alpha}\left|t-t^{\prime}\right|^{1-\beta}$.

THEOREM 2.6. - Any fixed point $\bar{u}=Q_{\kappa} \bar{u}, \bar{u} \in[0,1]$, satisfies $\bar{u} \in$ $C_{b}^{1, \alpha}(\mathbb{R})$.

Proof. - Provided $\alpha+\beta>1$ there is a similar argument as for (10) to show

$$
\frac{1}{|V|} \int_{V} \frac{\mathrm{d} \sigma_{v}}{\left|v_{1}-\kappa\right|^{1-\beta}} \leqslant \frac{4 \alpha C}{\alpha+\beta-1} .
$$

Any fixed point $\bar{u}, \bar{u} \in[0,1]$ is contained in $C_{b}^{\alpha}(\mathbb{R})$ by Lemma 2.4 . Hence, a bootstrap argument using the last lemma implies $\bar{u} \in C_{b}^{\beta}(\mathbb{R})$ for any $\beta \in(0,1)$. In particular, we can assume $\beta \geqslant 1-\alpha / 2$. Note, that $u=T_{\kappa} \circ \mathcal{F}[\bar{U}]$ is differentiable for $v_{1} \neq \kappa$ with

$$
\begin{aligned}
|\dot{u}(t, v)| & \leqslant \frac{1}{|V|}\left|v_{1}-\kappa\right|^{-1} \int_{-\infty}^{0} \mathrm{e}^{s}\left|F(\bar{u}(t))-F\left(\bar{u}\left(t+\left(v_{1}-\kappa\right) s\right)\right)\right| \mathrm{d} s \\
& \leqslant \frac{1}{|V|}\left|v_{1}-\kappa\right|^{\beta-1} \cdot 2 \operatorname{Lip} F|\bar{u}|_{C_{b}^{\beta}(\mathbb{R})} .
\end{aligned}
$$

We obtain by integration that $\bar{u}$ is uniformly Lipschitz continuous with Lipschitz constant bounded by $16 C \operatorname{Lip} F|\bar{u}|_{C_{b}^{\beta}(\mathbb{R})}$. Therefore, $\mathcal{F}[\bar{u}]$ is 
Lipschitz. Since $\dot{\bar{u}}=\bar{T}_{\kappa} \circ(\mathcal{F}[\bar{u}])^{\bullet}$ one final application of Lemma 2.4 gives $\bar{u} \in C_{b}^{1, \alpha}(\mathbb{R})$.

The theorem implies that any weak solution $u$ of (4), which is a priori only bounded, is a classical solution.

LEMMA 2.7. - Any fixed point $\bar{u}=Q_{\kappa} \bar{u}, \bar{u} \in[0,1]$, which is nonincreasing, is either strictly decreasing or constant.

Proof. - Let there be two points $t_{1}<t_{2}$ such that $\bar{u}\left(t_{1}\right)=\bar{u}\left(t_{2}\right)$. Then Eq. (29) reads

$$
\bar{u}\left(t_{i}\right)=\int_{\mathbb{R}} K_{\kappa^{*}}\left(s-t_{i}\right) F(\bar{u}(s)) \mathrm{d} s=\int_{\mathbb{R}} K_{\kappa^{*}}(s) F\left(\bar{u}\left(t_{i}+s\right)\right) \mathrm{d} s .
$$

Therefore $\bar{u}\left(t_{1}\right)-\bar{u}\left(t_{2}\right)=0$ implies

$$
\int_{\mathbb{R}} K_{\kappa^{*}}(s)\left(F\left(\bar{u}\left(t_{1}+s\right)\right)-F\left(\bar{u}\left(t_{2}+s\right)\right)\right) \mathrm{d} s=0 .
$$

Recall that $\bar{u}$ is nonincreasing and continuous by Lemma 2.6. Form the positivity of $K_{\kappa^{*}}(s)$ and the strict monotonicity of $F$ follows that $\bar{u}$ is a constant.

LEMMA 2.8. - The operator $\bar{T}_{\kappa}: L^{\infty}(\mathbb{R}) \rightarrow L^{\infty}(\mathbb{R})$, and hence $Q_{\kappa}$, is continuous in $\kappa$, as well as nonincreasing, if restricted to nonincreasing functions.

Proof. - Step 1 (Continuous dependence): Fix $\psi \in L^{\infty}(\mathbb{R})$ and let $-1<\kappa_{1}<\kappa_{2}<1$. Define $\kappa_{0}=\frac{1}{2}\left(\kappa_{1}+\kappa_{2}\right), \varepsilon:=\frac{1}{2}\left(\kappa_{2}-\kappa_{1}\right) \in(0,1)$ and $\delta:=\varepsilon+\sqrt[3]{\varepsilon}$. Then

$$
\left|\bar{T}_{\kappa_{1}}[\psi]-\bar{T}_{\kappa_{2}}[\psi]\right|_{L^{\infty}(\mathbb{R})} \leqslant|\psi|_{L^{\infty}(\mathbb{R})} \int_{\mathbb{R}}\left|K_{\kappa_{1}}(s)-K_{\kappa_{2}}(s)\right| \mathrm{d} s .
$$

For $s<0$ we obtain from the kernel representation

$$
|V|\left|K_{\kappa_{1}}(s)-K_{\kappa_{2}}(s)\right| \leqslant I_{1}(s)+I_{2}(s)+I_{3}(s),
$$

where

$$
I_{i, i=1,2}(s):=\int_{\kappa_{i}<v_{1}<\kappa_{0}+\delta} \frac{\mathrm{e}^{\frac{s}{v_{1}-\kappa_{i}}}}{v_{1}-\kappa_{i}} \mathrm{~d} \sigma_{v}
$$




$$
I_{3}:=\int_{v_{1}>\kappa_{0}+\delta}\left|\frac{\mathrm{e}^{\frac{s}{v_{1}-\kappa_{1}}}}{v_{1}-\kappa_{1}}-\frac{\mathrm{e}^{\frac{s}{v_{1}-\kappa_{2}}}}{v_{1}-\kappa_{2}}\right| \mathrm{d} \sigma_{v} .
$$

We estimate the integrals $I_{i, i=1,2}(s), i=1,2$, by

$$
\int_{-\infty}^{0} I_{i}(s) \mathrm{d} s=\int_{\kappa_{i}<v_{1}<\kappa_{0}+\delta} \mathrm{e}^{\frac{s}{v_{1}-\kappa_{i}}} \mathrm{~d} \sigma_{v} \leqslant\left|\left\{\kappa_{i}<v_{1}<\kappa_{0}+\delta\right\}\right| .
$$

For the last integral observe that

$$
\begin{aligned}
\left|\frac{\mathrm{e}^{\frac{s}{v_{1}-\kappa_{1}}}}{v_{1}-\kappa_{1}}-\frac{\mathrm{e}^{\frac{s}{v_{1}-\kappa_{2}}}}{v_{1}-\kappa_{2}}\right| & \leqslant \int_{\kappa_{1}}^{\kappa_{2}}\left(\frac{\mathrm{e}^{\frac{s}{v_{1}-\kappa}}}{\left(v_{1}-\kappa\right)^{3}}+\frac{\mathrm{e}^{\frac{s}{v_{1}-\kappa}}}{\left(v_{1}-\kappa\right)^{2}}\right) \mathrm{d} \kappa \\
& \leqslant 2 \varepsilon^{-2 / 3} \int_{\kappa_{1}}^{\kappa_{2}} \frac{\mathrm{e}^{\frac{s}{v_{1}-\kappa}}}{\left(v_{1}-\kappa\right)} \mathrm{d} \kappa,
\end{aligned}
$$

since $\kappa \in\left(\kappa_{1}, \kappa_{2}\right)$ and $v_{1}>\kappa_{0}+\delta$ implies $v_{1}-\kappa>\delta-\varepsilon=\sqrt[3]{\varepsilon}$. Therefore we obtain

$$
\int_{-\infty}^{0} I_{3}(s) \mathrm{d} s \leqslant\left(\varepsilon^{-2 / 3}+\varepsilon^{-1 / 3}\right) \int_{\kappa_{1}}^{\kappa_{2}} \int_{v_{1}>\kappa_{0}+\delta} \mathrm{e}^{\frac{s}{v_{1}-\kappa}} \mathrm{d} \sigma_{v} \mathrm{~d} \kappa \leqslant 2 \varepsilon^{1 / 3}|V| .
$$

Similar expressions are valid for $s>0$. Using (H3) we get

$$
\begin{aligned}
\int_{\mathbb{R}}\left|K_{\kappa_{1}}(s)-K_{\kappa_{2}}(s)\right| \mathrm{d} s & \leqslant \frac{2}{|V|}\left|\left\{\left|v_{1}-\kappa_{0}\right|<\delta\right\}\right|+4 \varepsilon^{1 / 3} \\
& \leqslant 2 C 2^{\alpha} \varepsilon^{\alpha / 3}+4 \varepsilon^{1 / 3} .
\end{aligned}
$$

Step 2 (Monotonicity): We show for any $\kappa_{1}<\kappa_{2}$, that $T_{\kappa_{1}} \geqslant T_{\kappa_{2}}$ on the subset of decreasing functions in $L^{\infty}(\mathbb{R})$. Let $\psi \in L^{\infty}(\mathbb{R})$ satisfy $\psi(s) \geqslant \psi\left(s^{\prime}\right)$ for all $s<s^{\prime}$. Since

$$
T_{\kappa_{i}}[\psi](t, v)=\frac{1}{|V|} \int_{-\infty}^{0} \mathrm{e}^{s} \psi\left(t+\left(v_{1}-\kappa_{i}\right) s\right) \mathrm{d} s,
$$

we obtain for the difference $\Delta:=T_{\kappa_{1}}[\psi]-T_{\kappa_{2}}[\psi]$

$$
\Delta(t, v)=\frac{1}{|V|} \int_{-\infty}^{0} \mathrm{e}^{s}\left(\psi\left(t+\left(v_{1}-\kappa_{1}\right) s\right)-\psi\left(t+\left(v_{1}-\kappa_{2}\right) s\right)\right) \mathrm{d} s
$$


which is nonnegative since $\kappa_{1}<\kappa_{2}$.

\subsection{The proof for type $A$}

For all $\kappa \in(-1,1)$ we define $c^{*}(\kappa)$ as the speed $c^{*}$ corresponding to the operator $Q_{\kappa}$ following the method of Weinberger. Lemma 2.8 implies that $c^{*}(\kappa)$ is lower semi-continuous and nonincreasing in $\kappa$.

LEMMA 2.9. - Type A implies $c^{*}(0)>0$.

Proof. - Consider $\kappa=0$, i.e., $Q=Q_{0}$ in the previous paragraph. For $c=0$ we consider the sequence (8) corresponding to a suitably chosen initial function $\phi$. Choose a $b \in(0,1)$. Define $\varepsilon:=F(b) / b-1>0$ and

$$
\phi(t):=b \cdot \begin{cases}1, & t<-\varepsilon^{-1}, \\ -\varepsilon s, & t \in\left[-\varepsilon^{-1}, 0\right] \\ 0, & t>0\end{cases}
$$

Since $F[\phi]=b(1+\varepsilon)$ on $\left(-\infty,-\varepsilon^{-1}\right]$ we obtain for any $t \in\left[-\varepsilon^{-1}, 0\right]$

$$
\begin{aligned}
& T_{0} \circ \mathcal{F}[\phi](t, v) \\
& \geqslant \frac{1}{|V|} b\left(-\varepsilon s+\varepsilon v_{1}\right)+\frac{1}{|V|} b \varepsilon \cdot \begin{cases}\left(1-v_{1}\right) \exp \left(\frac{-\varepsilon^{-1}-t}{v_{1}}\right), & v_{1}>0, \\
\left(-v_{1}\right) \exp \left(\frac{-t}{v_{1}}\right), & v_{1}<0 .\end{cases}
\end{aligned}
$$

Note $\int_{V} v_{1} \mathrm{~d} \sigma_{v}=0$ such that integration over $V$ yields

$$
Q_{0}[\phi]=\bar{T}_{0} \circ \mathcal{F}[\phi]>\phi \quad \text { for } t \in\left[-\varepsilon^{-1}, 0\right] .
$$

Note that $\phi$ is constant outside $\left[-\varepsilon^{-1}, 0\right]$. Let us recall that $Q_{0}[\phi]$ is nonincreasing and positive. Hence the inequality extends to all $t \in \mathbb{R}$. Furthermore, there exists a $\delta>0$ such that $Q_{0} \circ S_{\delta}[\phi]>\phi$. Hence, the sequence (8) satisfies

$$
w_{0}(0, t)=\phi, \quad w_{n+1}(0, t)=Q_{0} w_{n}>S_{-n \delta} \phi
$$

If $n \delta \geqslant \varepsilon^{-1}$ we can apply Proposition 2.2 to obtain $c=0<c^{*}(0)$.

In the next paragraph we show that $c^{*}(\kappa) \leqslant 0$ for speeds $\kappa$ close to 1 , see Lemma 2.11 below. Hence, the speed

$$
\kappa^{*}:=\sup \left\{\kappa \in(-1,1): c^{*}(\kappa)>0\right\}
$$


is well-defined. The lower semi-continuity of $c^{*}(\kappa)$ implies $c^{*}\left(\kappa^{*}\right) \leqslant 0$ and $\kappa^{*} \in(0,1)$.

Now Theorem 2.1 finishes the proof of Theorem 1.1, since we can conclude:

For all $\kappa<\kappa^{*}$ we have $c^{*}(\kappa)>0$ such that there is NO speed 0 front of $Q_{\kappa}$.

For any $\kappa \geqslant \kappa^{*}$ we have $c^{*}(\kappa) \leqslant 0$, i.e. there is a nonincreasing speed 0 front of $Q_{\kappa}$. Recall that a speed 0 front is equivalent to a solution of (9), which in turn is equivalent to a solution of (4). Furthermore, Lemma 2.7 provides the strict monotonicity.

\subsection{Minimal wave speed for subtangential source terms}

THEOREM 2.10. - Let $f$ be differentiable at 0 with $f^{\prime}(0)>0$. Then $F$ is differentiable at 0 with $F^{\prime}(0)>1$. If $F$ satisfies the subtangential property $F(z) \leqslant F^{\prime}(0) z$ then the minimal wave speed is given by

$$
\kappa^{*}=k\left(F^{\prime}(0)\right):=\inf _{\xi \in(0,1)} \frac{1}{\xi}\left(1-\frac{1}{A(\xi) F^{\prime}(0)}\right),
$$

where

$$
A(\xi)=\frac{1}{|V|} \int_{V} \frac{\mathrm{d} \sigma_{v}}{1-\xi v_{1}} .
$$

The speed function $k(\cdot)$ is continuous and strictly increasing with $k(1)=$ 0 and $k(\infty)=1$.

Note that $A(\xi)$ is smooth for $|\xi|<1$.

Proof. - We can find for every $\delta>0$ an $\varepsilon>0$ such that $F(z) \geqslant$ $(1-\delta) F^{\prime}(0) z$ for all $0 \leqslant z \leqslant \varepsilon$. We conclude from Theorem 2.3

$$
c^{*}(\kappa)=\inf _{\lambda>0} \frac{1}{\lambda} \log \int_{\mathbb{R}} F^{\prime}(0) \mathrm{e}^{-\lambda s} K_{\kappa}(s) \mathrm{d} s .
$$

In the following we show

$$
\begin{aligned}
\Lambda(\kappa, \lambda) & :=\int_{\mathbb{R}} \mathrm{e}^{\lambda s} K_{\kappa}(s) \mathrm{d} s \\
& = \begin{cases}\frac{1}{|V|} \int_{V} \frac{\mathrm{d} \sigma_{v}}{1+\lambda\left(v_{1}-\kappa\right)}, & \lambda \in\left[-\frac{1}{1-\kappa}, \frac{1}{1+\kappa}\right], \\
\infty, & \text { otherwise. }\end{cases}
\end{aligned}
$$


First observe that $\Lambda(\kappa, \lambda)=\bar{T}_{\kappa}\left[\mathrm{e}^{\lambda \cdot}\right](0)$. For any $\lambda \in\left(-\frac{1}{1-\kappa}, \frac{1}{1+\kappa}\right)$ the operator $\bar{T}_{\kappa}$ exhibits an eigenfunction $\bar{u}_{\lambda}=\mathrm{e}^{\lambda t}$ corresponding to the eigenvalue

$$
\Lambda(\kappa, \lambda)=\frac{1}{|V|} \int_{V} \frac{\mathrm{d} \sigma_{v}}{1+\lambda\left(v_{1}-\kappa\right)} .
$$

This proves the claim for such $\lambda$. If the integral is finite for $\lambda= \pm \frac{1}{1 \pm \kappa}$ it serves at least as an upper bound. We approximate $\mathrm{e}^{\lambda t}$ monotonically by $\min \left\{K, \mathrm{e}^{\lambda t}\right\}$ for increasing $K$ and obtain the reversed inequality. The latter idea also allows to prove the claim for $\lambda \notin\left[-\frac{1}{1-\kappa}, \frac{1}{1+\kappa}\right]$. In this case the lower bound $\bar{T}_{\kappa}\left[\min \left\{K, \mathrm{e}^{\lambda \cdot}\right\}\right](0)$ diverges for $K \rightarrow \infty$.

Using (14) we conclude for all $\kappa \geqslant 0$ that

$$
c^{*}(\kappa)>0 \quad \text { if and only if } \quad F^{\prime}(0) \inf _{\lambda>0} \Lambda(\kappa,-\lambda)>1
$$

Setting $\lambda(\xi):=-\frac{\xi}{1-\kappa \xi}, \xi \in(0,1)$, we obtain

$$
\Lambda\left(\kappa,-\frac{\xi}{1-\kappa \xi}\right)=(1-\kappa \xi) A(\xi)
$$

This yields

$$
c^{*}(\kappa)>0 \Leftrightarrow F^{\prime}(0) \inf _{\xi \in(0,1)}(1-\kappa \xi) A(\xi)>1 .
$$

Solving for the minimal $\kappa$ gives (11).

To get some information about the function $k(a), a \geqslant 1$, we need to examine $A(\xi)$ on $(0,1)$ first. Using the symmetry of $\sigma$ in Eq. (12) we obtain

$$
1 \leqslant A(\xi)=\frac{1}{|V|} \int_{V} \frac{\mathrm{d} \sigma_{v}}{1-\xi^{2} v_{1}^{2}} \leqslant \frac{1}{1-\xi^{2}}
$$

Hence,

$$
\inf _{\xi \in(0,1)} \frac{1}{\xi}\left(1-\frac{1}{a}\right)=\frac{a-1}{a} \leqslant k(a) \leqslant \inf _{\xi \in(0,1)} \frac{1}{\xi}\left(1-\frac{1-\xi^{2}}{a}\right) .
$$

The lower bound implies $k \geqslant 0$ and $\liminf _{a \rightarrow \infty} k(a) \geqslant 1$. We remark that the upper bound is the infimum over a convex function in $\xi$. Calculus 
yields

$$
k(a) \leqslant \begin{cases}2 \sqrt{a-1} / a, & a<2, \\ 1, & a \geqslant 2 .\end{cases}
$$

Hence, $k(1)=0, k \leqslant 1$, and, in particular, $\lim _{a \rightarrow \infty} k(a)=1$. Furthermore, $k$ is continuous at 1 . Now, let $a_{2}>a_{1} \geqslant a_{0}>1$. The definition of $k(a)$ implies

$$
\begin{aligned}
k\left(a_{2}\right) & \geqslant \frac{a_{1}}{a_{2}} k\left(a_{1}\right)+\inf _{\xi \in(0,1)} \frac{1}{\xi} \frac{a_{2}-a_{1}}{a_{2}} \\
& =k\left(a_{1}\right)+\frac{a_{2}-a_{1}}{a_{2}}\left(1-k\left(a_{1}\right)\right) .
\end{aligned}
$$

From (16) it follows that for $i=1,2$

$$
k\left(a_{i}\right)=\inf _{1-a_{0}^{-1} \leqslant \xi<1} \frac{1}{\xi}\left(1-\frac{1}{a_{i} A(\xi)}\right) .
$$

Hence,

$$
\begin{aligned}
k\left(a_{2}\right) & \leqslant \frac{a_{1}}{a_{2}} k\left(a_{1}\right)+\sup _{1-a_{0}^{-1} \leqslant \xi<1} \frac{1}{\xi} \frac{a_{2}-a_{1}}{a_{2}} \\
& =k\left(a_{1}\right)+\frac{a_{2}-a_{1}}{a_{2}}\left(\left(1-a_{0}^{-1}\right)^{-1}-k\left(a_{1}\right)\right) \\
& \leqslant k\left(a_{1}\right)+\frac{a_{2}-a_{1}}{a_{0}-1} .
\end{aligned}
$$

Together we obtain that $k$ is monotone and Lipschitz continues for $a>1$.

For the strict monotonicity it suffices by (17) to show $k<1$. To this end we derive an improved upper bound for $A(\xi), \xi$ close to 1 . With $\delta:=1-\xi$ we define $V_{\beta}:=\left\{v \in V: v_{1}>1-\delta^{\beta}\right\}$, where $\beta \in(0,1)$ will be chosen later. We estimate

$$
\frac{1}{1-\xi v_{1}} \leqslant \begin{cases}\frac{1}{1-(1-\delta)\left(1-\delta^{\beta}\right)} \leqslant \delta^{-\beta}, & \text { for all } v \notin V_{\beta} \\ \frac{1}{1-(1-\delta)}=\delta^{-1}, & \text { for all } v \in V_{\beta}\end{cases}
$$

Hence,

$$
A(\xi)=\frac{1}{|V|} \int_{V} \frac{\mathrm{d} \sigma_{v}}{1-\xi v_{1}}=\frac{1}{|V|} \int_{V \backslash V_{\beta}} \frac{\mathrm{d} \sigma_{v}}{1-\xi v_{1}}+\frac{1}{|V|} \int_{V_{\beta}} \frac{\mathrm{d} \sigma_{v}}{1-\xi v_{1}}
$$




$$
\begin{aligned}
& \leqslant \frac{1}{|V|} \int_{V \backslash V_{\beta}} \delta^{-\beta} \mathrm{d} \sigma_{v}+\frac{1}{|V|} \int_{V_{\beta}} \delta^{-1} \mathrm{~d} \sigma_{v} \\
& \leqslant \delta^{-\beta}+\delta^{-1} C \delta^{\alpha \beta} \quad \text { by (H3). }
\end{aligned}
$$

Setting $\beta:=\frac{1}{1+\alpha}$ we obtain

$$
A(\xi) \leqslant(1+C)(1-\xi)^{-\frac{1}{1+\alpha}}
$$

Recalling the definition of the minimal wave speed function we get

$$
\begin{aligned}
k(a) & =\inf _{\xi \in(0,1)} \frac{1}{\xi}\left(1-\frac{1}{a A(\xi)}\right) \leqslant \inf _{\xi \in(0,1)} \frac{1}{\xi}\left(1-\frac{(1-\xi)^{\frac{1}{1+\alpha}}}{a(1+C)}\right) \\
& \leqslant \frac{1-2 \delta_{a}}{1-\delta_{a}}<1-\delta_{a}, \quad \delta_{a}:=(2 a(1+C))^{-\frac{1+\alpha}{\alpha}} \in(0,1) .
\end{aligned}
$$

This shows that $k(a)<1$ for all $a \geqslant 1$.

The formulae of the minimal wave speed of subtangential nonlinearities is used to prove the following result for general nonlinearities.

LEMMA 2.11. - There is a $\bar{\kappa} \in(0,1)$ such that $c^{*}(\kappa) \leqslant 0$ for all $\kappa \in[\bar{\kappa}, 1)$.

Proof. - Step 1: Define $M:=\sup _{z \in(0,1)} F(z) / z$. From (H1) follows $1<M<\infty$. We use the minimal wave speed function $k(\cdot)$, defined above, and set $\bar{\kappa}:=k(M)$. Note that $\bar{\kappa} \in(0,1)$ by Theorem 2.10 . From the definition of $k(M)$ we know that there exists a $\lambda<0$ such that $M \Lambda(\bar{\kappa}, \lambda)=1$. Since $\bar{T}_{\bar{\kappa}} \mathrm{e}^{\lambda t}=\Lambda(\bar{\kappa}, \lambda) \mathrm{e}^{\lambda t}$ we deduce that $\phi_{\lambda}:=$ $\min \left\{1, \mathrm{e}^{\lambda t}\right\}$ is a super solution for $Q_{\bar{\kappa}}$.

Step 2: Let $\kappa \geqslant \bar{\kappa}$. Lemma 2.8 implies that $\phi_{\lambda}$ is also a super solution for $Q_{\kappa}$. Consider an arbitrary $\phi$ satisfying (7). Define for $c=0$ the sequence $\left\{w_{n}\right\}$ from (8). Since $Q_{\kappa}$ is monotone we obtain from $\phi \leqslant \phi_{\lambda}$ that $w_{n} \leqslant \phi_{\lambda}$ for any $n \in \mathbb{N}$. Hence, $w \leqslant \phi_{\lambda} \leqslant \mathrm{e}^{\lambda t}$ and thus $c=0 \geqslant c^{*}(\kappa)$ by Proposition 2.2. 


\section{THE TYPES B AND C}

\subsection{The finite problem}

Let us consider a Lipschitz nonlinearity $F$ satisfying $(\mathrm{H} 2)$ and $F(0)=$ $0, F(1)=1$. For $|\kappa|<1$ and $R>0$ we seek a solution $u(t, v)$ to the boundary value problem

$$
\begin{gathered}
\left(v_{1}-\kappa\right) \dot{u}+u=\frac{1}{|V|} F(\bar{u}), \quad|t|<R, \\
u(-R, v)=\frac{1}{|V|}, \quad v_{1}>\kappa, \\
u(+R, v)=0, \quad v_{1}<\kappa, \\
0<\bar{u}<1, \quad|t|<R .
\end{gathered}
$$

Let us define $W_{R}:=L^{\infty}([-R, R] ;[0,1])$. For any $\psi \in W_{R}$ we consider the linear problem to (19)-(21)

$$
\begin{gathered}
\left(v_{1}-\kappa\right) \dot{u}+u=\frac{1}{|V|} F(\psi), \quad|t|<R, \\
u(-R, v)=\frac{1}{|V|}, \quad v_{1}>\kappa, \\
u(+R, v)=0, \quad v_{1}<\kappa .
\end{gathered}
$$

Including the contribution from the boundary data we get from Section 2.1 the integral equation for finite domains

$$
\bar{u}=Q_{\kappa}^{R} \psi, \quad Q_{\kappa}^{R}:=B_{R} \circ Q_{\kappa} \circ E_{R} .
$$

Here

$$
E_{R}: W_{R} \rightarrow L^{\infty}(\mathbb{R}), \quad E_{R}[\psi](t):= \begin{cases}1, & t<-R \\ \psi(t), & |t| \leqslant R \\ 0, & t>R\end{cases}
$$

denotes an extension operator, and $B_{R}$ is the restriction onto the interval $[-R, R]$. Again, $Q_{\kappa}^{R}: W_{R} \rightarrow Y_{R}:=C^{\alpha}([-R, R] ;[0,1])$ is a monotone and continuous mapping, the estimates being uniform in $\kappa$.

A solution $u$ of (19)-(21) is equivalent to a fixed point $\bar{u}$ of $Q_{\kappa}^{R}: W_{R} \rightarrow$ $Y_{R}$. Once $\bar{u}$ is known, we can recover $u$ by $u=B_{R} \circ T_{\kappa} \circ \mathcal{F} \circ E_{R} \bar{u}$. In the following a function $\phi$ satisfying $\phi \leqslant Q_{\kappa}^{R} \phi$ (respectively $\phi \geqslant Q_{\kappa}^{R} \phi$ ) is called sub (respectively super) solution of $Q_{\kappa}^{R}$. 
THEOREM 3.1. - There exists a unique solution $u=u_{\kappa}^{R}$ of (19)-(22) satisfying

$$
\bar{u} \in Y_{R}, \quad\left(v_{1}-\kappa\right) \dot{u}, u \in L^{\infty}([-R, R] \times V) .
$$

In addition, $u$ (and hence $\bar{u}$ ) is strictly decreasing in t. Finally, if $\bar{u}_{0}$ is any sub (respectively super) solution of $Q_{\kappa}^{R}$ with $\bar{u}_{0} \in[0,1]$ then $\bar{u}_{0} \leqslant \bar{u}$ (respectively $\bar{u}_{0} \geqslant \bar{u}$ ).

Proof. - Step 1 (Existence): Since 0 and 1 are both fixed points of $F$, the constant functions $\bar{u}_{*} \equiv 0$ and $\bar{u}^{*} \equiv 1$ are sub and super solutions of $Q_{\kappa}^{R}$, respectively. Using the compactness and monotonicity of $Q_{\kappa}^{R}$ we construct a solution $\bar{u} \in Y_{R}$ by monotone approximation, starting with $\bar{u}_{*}$ or $\bar{u}^{*}$, respectively. Since $\bar{u}_{*} \leqslant \bar{u} \leqslant \bar{u}^{*}$ is necessarily satisfied, we get $\bar{u} \in[0,1]$.

Step 2 (Uniqueness): We use the sliding method.

Consider two solutions $u_{i}, i=1,2$, of (19)-(21). Equivalently, we can say $\bar{u}_{i}=Q_{\kappa}^{R} \bar{u}_{i}$. Instead of (22) we only require $\bar{u}_{i} \in[0,1]$. We extend $\bar{u}_{i}$ naturally on $\mathbb{R}$ by just not restricting it, i.e. setting $\bar{u}_{i}=\bar{T}_{\kappa} \circ \mathcal{F} \circ E_{R} \bar{u}_{i}$ for all $|t|>R$. Note $\bar{u}_{i} \in C_{b}^{\alpha}(\mathbb{R})$ by Lemma 2.4. Let $\phi_{0}=\bar{T}_{\kappa}[H(-\cdot)]$, $H$ being the Heaviside function. The positivity of the kernel $K_{\kappa}$ ensures $\phi_{0} \in(0,1)$. From (22) and (H2) we obtain

$$
\phi_{0}(\cdot+R)=\bar{T}_{\kappa} \circ \mathcal{F} \circ E_{R} 0 \leqslant \bar{u}_{i} \leqslant \bar{T}_{\kappa} \circ \mathcal{F} \circ E_{R} 1=\phi_{0}(\cdot-R) .
$$

Hence $\bar{u}_{i}$ must satisfy (22). Since $\bar{u}_{i}$ is continuous, the difference $\Delta:=$ $\bar{u}_{1}-\bar{u}_{2}$ is continuous. We can assume that there exists a point $t_{1},\left|t_{1}\right| \leqslant R$ such that $\Delta\left(t_{1}\right)>0$. Otherwise interchange the index or $\left.\Delta\right|_{[-R, R]} \equiv 0$ and we are ready.

The shift operator is continuous on $C_{b}^{\alpha}(\mathbb{R})$ such that $\Delta_{s}(t)=\bar{u}_{1}(t+$ $s)-\bar{u}_{2}(t)$ is again continuous for all $s \in \mathbb{R}$. We assumed $\Delta_{0}\left(t_{1}\right)>0$ and get from (23) $\Delta_{2 R} \leqslant 0$. Restricting our attention to the finite interval $[-R,+R]$ we obtain, that there must exist a shift $s_{0} \in(0,2 R]$ such that $\left.\Delta_{s_{0}}\right|_{[-R, R]} \leqslant 0$ with equality for some $t_{0},\left|t_{0}\right| \leqslant R$. Since $\bar{T}_{\kappa}$ is a convolution we obtain

$$
\begin{gathered}
\bar{u}_{i}(t+s)=\bar{T}_{\kappa} \Psi_{i, s}(t), \\
\Psi_{i, s}(t):= \begin{cases}1, & t<-R-s, \\
F\left(\bar{u}_{i}(t+s)\right), & -R-s \leqslant t \leqslant R-s, \\
0, & t>R-s,\end{cases}
\end{gathered}
$$


such that $\Delta_{s}=\bar{T}_{\kappa}\left(\Psi_{1, s}-\Psi_{2,0}\right)$. From (22), (H2) and $\left.\Delta_{s_{0}}\right|_{[-R, R]} \leqslant 0$ follows $\Psi_{1, s_{0}}-\Psi_{2,0} \leqslant 0$. Since $\bar{T}_{\kappa}$ is a positive operator $\Delta_{s_{0}}\left(t_{0}\right)=0$ implies $\Psi_{1, s_{0}}-\Psi_{2,0} \equiv 0$. In particular it holds for $t \in\left(R-s_{0}, R\right)$

$$
0=\Psi_{1, s_{0}}(t)-\Psi_{2,0}(t)=0-F\left(\bar{u}_{2}(t)\right),
$$

hence, $\bar{u}_{2} \equiv 0$ on $\left(R-s_{0}, R\right)$. Since this contradicts (22) we proved uniqueness.

Note that the method of monotone approximation from step 1 implies that the unique $\bar{u}$ is sandwiched between any pair of sub and super solutions of $Q_{\kappa}^{R}$ with values in $[0,1]$.

Step 3 (Monotonicity): Since $\bar{T}_{\kappa}$ is a convolution with positive kernel, it leaves invariant the space of pointwise nonincreasing/nondecreasing functions on $\mathbb{R}$. By $(\mathrm{H} 2)$, recall also the precise form of boundary condition, it follows that $Q_{\kappa}^{R}$ leaves invariant the space of nonincreasing functions in $W_{R}$. Recall that in step 1 the solution $\bar{u}$ is constructed by a monotone approximation starting with the constant function 0 or 1 . Hence, $\bar{u}$ must be nonincreasing in $t$. The strict monotonicity is proven by another application of the sliding method similar to step 2 .

THEOREM 3.2. - The solution $u=u_{\kappa}^{R}$ of (19)-(22) is strictly decreasing in $\kappa$. Furthermore, $\bar{u}_{\kappa}^{R}$ is continuous in $\kappa$.

For the continuity statement it is important to consider $\bar{u}$ instead of $u$ itself, because it enjoys better regularity properties, described above. However, no $C^{0}$ estimate for $u$ can be expected, since the hyperbolic boundary conditions generate a jump in the dependence of $u$ on $v$ if the value of $v_{1}$ crosses $\kappa$.

Proof. - Step 1 (Continuous dependence): Note that the compactness property $Q_{\kappa}^{R}: W_{R} \rightarrow Y_{R}$ holds uniformly in $\kappa$. Since we established already the uniqueness of solutions $\bar{u} \in(0,1)$ for all $\kappa$, the continuous dependence follows classical steps: To a given sequence $\kappa_{i} \rightarrow \kappa$ consider the unique solutions $\bar{u}_{i}:=\bar{u}_{\kappa_{i}}^{R}$. Going over to a subsequence if needed, the $\bar{u}_{i}$ converge uniformly to a function $\bar{u} \in Y_{R}$. But this $\bar{u}$ solves $\bar{u}=Q_{\kappa}^{R} \bar{u}$, i.e., represents a solution to the speed $\kappa$. Uniqueness ensures $\bar{u}=\bar{u}_{\kappa}^{R}$.

Step 2: In step 2 of the proof of Lemma 2.8 we showed that $T_{\kappa} \psi$ is a nonincreasing function of $\kappa$, provided $\psi \in L^{\infty}(\mathbb{R})$ is nonincreasing. By (H2) follows

$$
\Delta(t, v):=B_{R} \circ T_{\kappa_{1}} \circ \mathcal{F} \circ E_{R} \psi-B_{R} \circ T_{\kappa_{2}} \circ \mathcal{F} \circ E_{R} \psi \geqslant 0 .
$$


Integrating over $V$ gives only the non-strict version of the claim. To obtain the strict inequality we observe that for any $s>R$ we have $\mathcal{F} \circ E_{R}[\psi](-s)=1>0=\mathcal{F} \circ E_{R}[\psi](s)$. This allows us to improve the inequality above for $v \in I:=\left[\kappa_{1}+\delta, \kappa_{2}-\delta\right], \delta:=\frac{\kappa_{2}-\kappa_{1}}{4}>0$. For all $|t|<R$ we have $M:=\sup _{v \in I} \max \left\{\frac{R+t}{\left|v_{1}-\kappa_{1}\right|}, \frac{R-t}{\left|v_{1}-\kappa_{2}\right|}\right\}<\frac{2 R}{\delta}$, such that

$$
\begin{aligned}
\Delta(t, v)= & \frac{1}{|V|} \int_{-\infty}^{0} \mathrm{e}^{s}\left(F\left(E_{R} \psi\left(t-\left|\left(v_{1}-\kappa_{1}\right) s\right|\right)\right)\right. \\
& -F\left(E_{R} \psi\left(t+\left|\left(v_{1}-\kappa_{2}\right) s\right|\right)\right) \mathrm{d} s \\
\geqslant & \frac{1}{|V|} \int_{-\infty}^{-M} \mathrm{e}^{s} \mathrm{~d} s=\frac{1}{|V|} \mathrm{e}^{-M}>0 .
\end{aligned}
$$

Since $\sigma_{v}$ is a positive measure we obtain $\bar{\Delta}>0$.

Step 3: Since $\bar{u}_{\kappa_{1}}^{R}$ is the fixed point of $Q_{\kappa_{1}}^{R}$, we know from Theorem 3.1 that it is decreasing in $t$. Applying the results of steps 2 and 3 we find

$$
\bar{u}_{\kappa_{1}}^{R}=Q_{\kappa_{1}}^{R} \bar{u}_{\kappa_{1}}^{R}>Q_{\kappa_{2}}^{R} \bar{u}_{\kappa_{1}}^{R},
$$

implying $\bar{u}_{\kappa_{2}}^{R}<\bar{u}_{\kappa_{1}}^{R}$ by Theorem 3.1. Since $u_{\kappa_{i}}^{R}=T_{\kappa} \circ \mathcal{F} \circ E_{R} \bar{u}_{\kappa_{i}}^{R}$ depends monotonically on $\bar{u}_{\kappa_{i}}^{R}$, we finally get $u_{\kappa_{2}}^{R}<u_{\kappa_{1}}^{R}$.

COROLlary 3.3. - Let $\theta_{0} \in[\theta, 1)$. There exist constants $R_{0}(\theta)>0$, $-1<\underline{\kappa}\left(\theta_{0}\right)<0<\bar{\kappa}<1$, such that for any $R>R_{0}$ there is a unique speed $\kappa_{R} \in(\underline{\kappa}, \bar{\kappa})$ such that $\bar{u}_{\kappa_{R}}^{R}(0)=\theta_{0}$.

Proof. - Step 1 (Lower bound for $\kappa$ ): Let $R>0$ and $\phi_{0}^{\kappa}:=T_{\kappa} \circ$ $H(-\cdot)$, where $H$ denotes the Heaviside function. Choose $z \in\left(\theta_{0}, 1\right)$ and define $v:=z / F(z) \in(0,1)$. The function $\bar{u}_{0} \equiv z$ satisfies $Q_{\kappa}^{R} \bar{u}_{0}(t) \geqslant$ $F(z) \bar{\phi}_{0}^{\kappa}(t-R)$. Therefore $\bar{u}_{0} \leqslant Q_{\kappa}^{R} \bar{u}_{0}$ is implied by the inequality

$$
\bar{\phi}_{0}^{\kappa}(t) \geqslant v \quad \text { for all } t<0 .
$$

For $t<0$ we have $\phi_{0}^{\kappa}\left(t, v_{1}>\kappa\right) \equiv 1 /|V|$ and $\phi_{0}^{\kappa}\left(t, v_{1}<\kappa\right) \geqslant 0$, such that by $(\mathrm{H} 3)$

$$
\bar{\phi}_{0}^{\kappa}(t) \geqslant 1-\frac{1}{|V|}\left|\left\{v \in V: v_{1}<\kappa\right\}\right| \geqslant 1-C(1+\kappa)^{\alpha} .
$$


Hence, for all $\kappa<\underline{\kappa}:=((1-v) / C)^{1 / \alpha}-1<0$, recall $C \geqslant 1$, we obtain

$$
\bar{u}_{\kappa}^{R}(0) \geqslant \bar{u}_{0}(0)=z>\theta_{0} .
$$

Step 2 (Upper bound for $\kappa)$ : We take the speed $\bar{\kappa}:=k\left(\sup _{z \in(0,1)} F(z) /\right.$ $z$ ) from Lemma 2.11. There we showed that there is a $\lambda<0$ such that $\min \left\{1, \mathrm{e}^{\lambda t}\right\}$ is a super solution for all $Q_{\kappa}, \kappa \geqslant \bar{\kappa}$. Since $E_{R}\left[\mathrm{e}^{\lambda(++R)}\right](t) \leqslant$ $\mathrm{e}^{\lambda(t+R)}$ we deduce that $\mathrm{e}^{\lambda(t+R)}$ is a super solution for $Q_{\kappa}^{R}, \kappa \geqslant \bar{\kappa}$. This implies

$$
\bar{u}_{\kappa}^{R}(0) \leqslant \mathrm{e}^{\lambda R} .
$$

Hence, $\bar{u}_{\kappa}^{R}(0)<\theta_{0}$ for all $\kappa \geqslant \bar{\kappa}$ and $R>R_{0}:=|\lambda|^{-1} \ln \theta_{0}^{-1}$.

Step 3: Using Theorem 3.2 and the previous two steps we can apply the intermediate value theorem on the continuous function $\kappa \mapsto \bar{u}_{\kappa}^{R}(0)$. This yields the existence of the unique speed $\kappa_{R} \in(\underline{\kappa}, \bar{\kappa})$.

\subsection{Constructing the front}

We use the solutions $u_{\kappa_{R}}^{R}$ constructed in Theorem 3.1 for increasing values of $R$ and corresponding speeds $\kappa_{R} \in(\underline{\kappa}, \bar{\kappa})$ given by Corollary 3.3 choosing $\theta_{0}=\theta$. Extract a subsequence $R_{i} \rightarrow \infty$ such that $\kappa_{R_{i}} \rightarrow \kappa^{*} \in$ $[\underline{\kappa}, \bar{\kappa}]$. The total densities satisfy

$$
\begin{gathered}
\bar{u}_{\kappa_{R_{i}}}^{R_{i}} \in C^{\alpha}([-R, R] ;[0,1]) \text { and strictly decreasing in } t, \\
\bar{u}_{\kappa_{R_{i}}}^{R_{i}}(0)=\theta, \\
\bar{u}_{\kappa_{R_{i}}}^{R_{i}}=Q_{\kappa_{R_{i}}}^{R_{i}}\left[\bar{u}_{\kappa_{R_{i}}}^{R_{i}}\right] .
\end{gathered}
$$

Choosing again a subsequence if necessary, $\bar{u}_{\kappa_{R_{i}}}^{R_{i}}$ converge uniformly on bounded sets to a function $\bar{u}$, which satisfies

$$
\begin{gathered}
\bar{u} \in C^{\alpha}(\mathbb{R} ;[0,1]) \text { and nonincreasing in } t, \\
\bar{u}(0)=\theta, \\
\bar{u}=Q_{\kappa^{*}}[\bar{u}] .
\end{gathered}
$$

Theorem 2.6 implies $\bar{u} \in C_{b}^{1, \alpha}(\mathbb{R})$. We show in the remainder of this paragraph that the function $\bar{u}$, constructed above, is indeed a solution 
of (9). This amounts to prove that $\bar{u}$ attains the asymptotic boundary conditions. Recall that $\kappa^{*} \in[\underline{\kappa}, \bar{\kappa}]$. At the end of this paragraph we will even show that there cannot be any solution of (9) with $\kappa>\bar{\kappa}$.

Lemma 2.7 implies that $\bar{u}$ is either strictly decreasing or equal to a constant, which must be $\theta$ by (28). From (27) follows that $\bar{u}$ has asymptotic limits

$$
\begin{array}{cl}
\Psi_{ \pm}:=\lim _{t \rightarrow \pm \infty} \bar{u}(t) & \text { satisfying } 0 \leqslant \Psi_{+}<\theta<\Psi_{-} \leqslant 1 \\
& \text { or } \Psi_{+}=\theta=\Psi_{-} .
\end{array}
$$

Furthermore, $\Psi_{-}$and $\Psi_{+}$have to be fixed points of the mapping $F$. The proof is given by contradiction. Assume $\Psi_{-}=b \in(\theta, 1)$. There exist $b^{\prime} \in(\theta, b)$ and $t^{\prime} \in \mathbb{R}$ such that $F\left(b^{\prime}\right)>b$ and $\bar{u}(t)>b^{\prime} H\left(t^{\prime}-t\right), H$ being the Heaviside function. It follows from (29)

$$
\begin{aligned}
\bar{u}(t) & \geqslant Q_{\kappa^{*}}\left[b^{\prime} H\left(t^{\prime}-t\right)\right]=F\left(b^{\prime}\right) T_{\kappa^{*}}\left[H\left(t^{\prime}-t\right)\right] \\
& \geqslant F\left(b^{\prime}\right)\left(1-\exp \left(-\frac{t^{\prime}-t}{1+\kappa^{*}}\right)\right) .
\end{aligned}
$$

Hence, $\Psi_{-} \geqslant F\left(b^{\prime}\right)>b$, which is impossible. Type $\mathrm{C}$ admits a similar argument for $\Psi_{+}$.

We summarise:

Type B: Either $\bar{u} \equiv \theta$ or $\Psi_{-}=1, \Psi_{+} \in[0, \theta)$.

Type C: Either $\bar{u} \equiv \theta$ or $\Psi_{-}=1, \Psi_{+}=0$.

LEMMA 3.4. - If $\bar{u} \equiv \theta$ then there exists a travelling front, not faster than $\kappa^{*}$, connecting 1 and $\theta$. Hence, type A implies $\kappa^{*}>0$.

Proof. - Recall the method of construction leading to a solution on the whole line, which was prescribed in the beginning of this section. But now we choose $\theta_{0} \in(\theta, 1)$. For any $i \in \mathbb{N}$ there is a unique solution of

$$
\bar{u}_{\kappa_{R_{i}}^{\prime}}^{R_{i}}=Q_{\kappa_{R_{i}}^{\prime}}^{R_{i}}\left[\bar{u}_{\kappa_{R_{i}}^{\prime}}^{R_{i}}\right], \quad \bar{u}_{\kappa_{R_{i}}^{\prime}}^{R_{i}}(0)=\theta_{0}
$$

From Theorem 3.2 and Corollary 3.3 we know $\underline{\kappa}\left(\theta_{0}\right)<\kappa_{R_{i}}^{\prime}<\kappa_{R_{i}}$ and $\bar{u}_{\kappa_{R_{i}}^{\prime}}^{R_{i}}>\bar{u}_{\kappa_{R_{i}}}^{R_{i}}$. For a subsequence the speeds $\kappa_{R_{i}}^{\prime}$ will converge to a speed $\kappa^{\prime} \leqslant \kappa^{*}$ while the total densities converge uniformly on bounded sets to a function $\bar{u}^{\prime} \geqslant \bar{u} \equiv \theta$ satisfying

$$
\bar{u}^{\prime} \in C^{\alpha}(\mathbb{R} ;[\theta, 1]) \text { and nonincreasing in } t,
$$




$$
\begin{gathered}
\bar{u}^{\prime}(0)=\theta_{0}, \\
\bar{u}^{\prime}=Q_{\kappa^{\prime}}\left[\bar{u}^{\prime}\right] .
\end{gathered}
$$

Recall that $F$ has no fixed points in $(\theta, 1)$. Hence, $\bar{u}^{\prime}$ is a travelling front with asymptotic values 1 and $\theta$ at $-\infty$ and $+\infty$, respectively. Hence, $\bar{u}^{\prime}$ is even strictly decreasing by Lemma 2.7 . The nonlinearity restricted to $[\theta, 1]$ is of type $\mathrm{A}$, such that Theorem 1.1 implies $0<\kappa^{\prime} \leqslant \kappa^{*}$.

Lemma 3.5. - Consider type B. Then $\Psi_{-}=1$ implies $\kappa^{*}>0$.

Proof. - Assume $\kappa^{*} \leqslant 0$. We apply the sliding method in order to compare $\bar{u}$ and the test function $\phi$ from the proof of Lemma 2.9. Choosing a $b \in(\theta, 1)$ the proof will also apply for nonlinearities of type B. Thus, $\phi$ is a sub solution to $Q_{0}$, and hence for $Q_{\kappa^{*}}$ by Lemma 2.8. Recall that $\bar{u}$ solves $\bar{u}=Q_{\kappa^{*}}[\bar{u}]$. Furthermore, since $\Psi_{-}=1$ Lemma 2.7 shows that $\bar{u}$ is strictly decreasing. Since $\phi(t)=b$ for $t<-\varepsilon^{-1}$ and $\phi(t)=0$ for $t \geqslant 0$ we have $\bar{u}(t)>\phi(t)$ for large values of $|t|$. This remains true for any finite shift of the function $\phi$. If we shift $\phi$ to the left we will achieve $S_{M} \phi<\bar{u}$ for some large $M>0$. From $\bar{u}(0)=\theta<b$ we know that $S_{-\varepsilon^{-1}} \phi(0)>\bar{u}(0)$. Hence there is a shift $S_{s_{0}}$ such that $S_{s_{0}} \phi \leqslant \bar{u}$ with equality at some finite point $t_{1}$. Since $\phi$ is a subsolution we obtain

$$
\begin{aligned}
0 & \geqslant Q_{\kappa^{*}}[\bar{u}]\left(t_{1}\right)-Q_{\kappa^{*}}\left[S_{s_{0}} \phi\right]\left(t_{1}\right) \\
& =\int_{\mathbb{R}} K_{\kappa^{*}}\left(s-t_{1}\right)\left(F(\bar{u}(s))-F\left(S_{s_{0}} \phi(s)\right)\right) \mathrm{d} s .
\end{aligned}
$$

From $S_{s_{0}} \phi \leqslant \bar{u}$ follows by (H2) that the integrand is nonnegative, hence vanishes. Thus, $K_{K^{*}}>0$ implies $S_{S_{0}} \phi=\bar{u}$, contradicting $\Psi_{-}=1$.

Lemma 3.6. - Assume type $B$ or $C$. If $\kappa^{*}>0$ then $\Psi_{+}=0$.

Proof. - Consider the following linear problem

$$
\begin{gathered}
(v-\kappa) \dot{u}+u=\frac{1}{|V|} \bar{u}, \quad t \in(0, R), \\
u(0, v)=\frac{1}{|V|}, \quad v>\kappa, \\
u(+R, v)=0, \quad v<\kappa .
\end{gathered}
$$

A solution is equivalent to a fixed point of $\tilde{Q}_{\kappa}^{R}:=\tilde{B}_{R} \circ \bar{T}_{\kappa} \circ \tilde{E}_{R}$, where $\tilde{B}_{R}$ restricts functions onto $[0, R]$, and $\tilde{E}_{R}$ extends functions by 1 on $(-\infty, 0)$ and by 0 on $(R, \infty)$. For $\kappa=\kappa_{R_{i}}$ and $R=R_{i}$ the function $\tilde{B}_{R} \bar{u}_{\kappa_{R_{i}}}^{R_{i}}$ is a sub solution. This follows immediately from $F(z) \leqslant z, z \in[0, \theta]$ if $F$ is of 
type $\mathrm{B}$ or $\mathrm{C}$. On the other hand, for any positive $\kappa$ and $R$ we can choose $\phi_{\lambda}(t):=\min \left\{1, \mathrm{e}^{\lambda t}\right\}$ as a super solution, provided that $\Lambda(\kappa, \lambda) \leqslant 1$ and $\lambda \in\left(-\frac{1}{1-\kappa}, 0\right)$, see also Section 2.3. To allow for negative values of $\lambda$ the positivity of $\kappa^{*}$ comes into play. Since $\kappa^{*}>0$, there is an $i_{0}$ such that

$$
\kappa_{R_{i}}>\kappa_{0}:=\frac{1}{2} \kappa^{*}>0 \text { for all } i>i_{0}
$$

From the proof of Theorem 2.10 in Section 2.3 we know for all $\xi \in(0,1)$

$$
\Lambda\left(\kappa,-\frac{\xi}{1-\kappa \xi}\right)=(1-\kappa \xi) A(\xi) \quad \text { with } A(\xi) \leqslant\left(1-\xi^{2}\right)^{-1}
$$

Setting $\lambda_{0}:=-\kappa_{0}$ we obtain for all $\kappa \geqslant \kappa_{0}$

$$
\Lambda\left(\kappa, \lambda_{0}\right) \leqslant \frac{1+\kappa \kappa_{0}}{\left(1+\kappa \kappa_{0}\right)^{2}-\kappa_{0}^{2}} \leqslant \frac{1+\kappa \kappa_{0}}{1+\kappa \kappa_{0}+\left(\kappa-\kappa_{0}\right) \kappa_{0}} \leqslant 1,
$$

showing that $\phi_{\lambda_{0}}$ is a super solution. Hence, for any $i>i_{0}$ Theorem 3.1 yields the existence of a solution with data $\kappa=\kappa_{R_{i}}$ and $R=R_{i}$. Furthermore, we obtain the uniform inequality

$$
\bar{u}_{\kappa R_{i}}^{R_{i}}(t)<\phi_{\lambda_{0}}(t) \leqslant \mathrm{e}^{\lambda_{0} t} \quad \text { for all } t \in\left[0, R_{i}\right]
$$

This implies $\bar{u} \leqslant \mathrm{e}^{\lambda_{0} t}$ for all $t \in \mathbb{R}_{+}$, proving $\Psi_{+}=0$.

Now we conclude the proof of Theorem 1.2.

Type B: For all possible values of $\Psi_{ \pm}$we conclude from Lemmas 3.4 and 3.5 that $\kappa^{*}>0$. Hence, $\Psi_{+}=0$ by Lemma 3.6 and thus $\Psi_{-}=1$ by (30).

Type C: By (30) it suffices to exclude the case $\bar{u} \equiv \theta$. Here Lemma 3.4 implies $\kappa^{*}>0$. But then $\Psi_{+}=0$ by Lemma 3.6, contradicting $\bar{u} \equiv \theta$.

It remains to show that there is no solution $(\kappa, \bar{u})$ of $(9)$ for any $\kappa>\bar{\kappa}$. Recall from Lemma 2.11 the definition $\bar{\kappa}:=k(M)$, where $M=$ $\sup _{z \in(0,1)} F(z) / z>1$. For any $\kappa \in(\bar{\kappa}, 1)$ we can find by Theorem 2.10 a slope $M^{\prime}>M$ such that $k\left(M^{\prime}\right)=\kappa$. Since $M^{\prime}>M$ there is a $\theta_{1} \in(0, \theta)$ such that $F(z) \leqslant \theta_{1}+M^{\prime}\left(z-\theta_{1}\right)$ for all $z \in\left[\theta_{1}, 1\right]$. As in the proof of Lemma 2.11 we can find a $\lambda^{\prime}<0$ such that $M^{\prime} \Lambda\left(\kappa, \lambda^{\prime}\right) \leqslant 1$. Hence, $\phi:=\min \left\{1, \theta_{1}+\mathrm{e}^{\lambda^{\prime} t}\right\}$ is a super solution for $Q_{\kappa}$. Assuming the existence of a solution $(\kappa, \bar{u})$ of (9) we can apply the sliding method in order to compare $\bar{u}$ and $\phi$. Arguing similar to the proof of Lemma 3.5 we deduce that $\bar{u}$ must be a finite shift of $\phi$. Hence, $\bar{u}$ cannot be a solution of (9). 


\section{REFERENCES}

[1] Aronson. D.G., The asymptotic speed of propagation of a simple epidemic, in: Fitzgibbon W.E., Walker H.F. (Eds.), Nonlinear Diffusion, Res. Notes Math., Vol. 14, Pitman, 1977, pp. 1-23.

[2] Aronson D.G., Weinberger H.F., Nonlinear diffusion in population genetics, combustion, and nerve pulse propagation, in: Goldstein J. (Ed.), Partial Differential Equations and Related Topics, Lect. Notes Math., Vol. 446, Springer, 1975, pp. 549.

[3] Aronson D.G., Weinberger H.F., Multidimensional nonlinear diffusion arising in population genetics, Adv. Math. 30 (1978) 33-76.

[4] Berestycki H., Larrouturou B., A semilinear elliptic equation in a strip arising in a two-dimensional flame propagation model, J. Reine Angew. Math. 396 (1989) $14-40$.

[5] Berestycki H., Nirenberg L., Travelling fronts in cylinders, Ann. Inst. Henri Poincaré, Anal. Non Linéaire 9 (5) (1992) 497-572.

[6] Diekmann O., Run for your life. A note on the asymptotic speed of propagation of an epidemic, J. Differential Equations 33 (1979) 58-73.

[7] Fisher R.A., The advance of advantageous genes, Ann. Eugenics 7 (1937) 355-369.

[8] Golse F., Lions P.-L., Perthame B., Sentis R., Regularity of the moments of the solution of a transport equation, J. Functional Analysis 79 (1) (1988) 110-125.

[9] Hadeler K.P., Travelling fronts for correlated random walks, Can. Appl. Math. Q. 2 (1) (1994) 27-43.

[10] Hadeler K.P., Reaction transport systems in biological modelling, in: Mathematics Inspired by Biology, Lect. Notes Math., Vol. 1714, Springer, 1999.

[11] Kolmogorov A.N., Petrovsky I.G., Piscounov N.S., Étude de l'équation de la diffusion avec croissance de la quantité de matière et son application à un problème biologique, Bull. Univ. État Moscou, Ser. Int., Sect. A, Math. et Mecan. 1 (6) (1937) 1-25; English Transl. in: Pelcé R. (Ed.), Dynamics of Curved Fronts, Academic Press, 1988, p. 105-130.

[12] Othmer H.G., Dunbar S.R., Alt W., Models of dispersal in biological systems, J. Math. Biol. 26 (3) (1988) 263-298.

[13] Schumacher K., Travelling-front solutions for integro-differential equations. I, J. Reine Angew. Math. 316 (1980) 54-70.

[14] Thieme H.R., Asymptotic estimates of the solutions of nonlinear integral equations and asymptotic speeds for the spread of populations, J. Reine Angew. Math. 306 (1979) 94-121.

[15] Weinberger H.F., Asymptotic behavior of a model in population genetics, in: Chadam J.M. (Ed.), Nonlinear Partial Differential Equations and Applications, Lect. Notes Math., Vol. 648, Springer, 1978, pp. 47-96.

[16] Weinberger H.F., Long-time behavior of a class of biological models, SIAM J. Math. Anal. 13 (3) (1982) 353-396. 